M. Moh a m madian ${ }^{a}$, O.Pourmehran ${ }^{b}, P . J u^{c}$

\title{
AN ITERATIVE APPROACH FOR OBTAINING NONLINEAR FREQUENCY OF A CONSERVATIVE OSCILLATOR WITH STRONG NONLINEARITIES
}

\author{
${ }^{a}$ Department of Mechanical Engineering, Kordkuy center, \\ Gorgan branch, Islamic Azad University, Kordkuy, Iran. \\ ${ }^{b}$ Young Researchers and Elite Club, Gorgan Branch, Islamic Azad University, Gorgan, Iran. \\ ${ }^{c}$ School of Mathematics and Statistics, Taishan University, Taian, PR China. \\ * Corresponding author: P.O.B. 48811644479 Kordkuy,mo.mohammadyan@gmail.com
}

\begin{abstract}
An iterative procedure is suggested for obtaining the higher-order approximate solutions of a conservative system comprising an oscillator with cubic and quintic restoring force function. The proposed method is similar to the traditional harmonic balance methods but unlike them the obtained from the previous step errors are considered in the present step to increase the accuracy of the solution. A comparison of results with those obtained by exact solution and other approximate analytical techniques confirms an accuracy of the method. It is shown that the achieved approximate solutions are valid for both small and large amplitudes of oscillation and can meet the exact solutions with a high level of accuracy in the lower-order of approximations. Furthermore, using the obtained analytical solutions, the effect of cubic and quintic terms on the frequency is discussed.
\end{abstract}

Key words: harmonic balance method, Duffing oscillator, analytical solution, frequency.

\section{Introduction.}

A large bunch of the mechanical systems are involved with nonlinear governing equation of motion which solving these equations is an important issue for the sake of their nonlinear behavior [1-3]. Transverse vibrations of a beam with large amplitude and implementation of nonlinear springs in a mechanical system are two examples which can be derived as a nonlinear ordinary differential equation. Recently, many different methods have been developed to obtain the approximate solutions of such problems. Some of them are: harmonic balance method [4-6], energy balance method [7, 8], Hamiltonian approach [9], He's amplitude-frequency formulation [10] and variational iteration method [11]. In these methods, unlike the classical perturbation method, the presence of small parameter is not required.

Duffing equation is a well-known nonlinear differential equation which is composed of third and fifth orders of nonlinearities. Many applications of this equation can be found in some engineering systems such as free vibrations of a restrained uniform beam including a lumped mass in its middle region [12], the nonlinear dynamics of slender elastic, and the Pochhammer- Chree (PC) equation $[13,14]$.

A combination of homotopy analysis method and Pade technique was employed by Pirbodaghi et al. [15] to obtain the analytical approximate solution of the cubic-quintic Duffing equation. Ganji et al. [8] considered different parameters and applied energy balance technique on the approximate frequencies of the mentioned equation. In another research, Newton's method and harmonic balance technique were coupled by Lai et al. [16] for solving higher-order approximations of Duffing oscillators with cubic-quintic nonlinear restoring force. They showed that their results are valid for both small and large amplitudes. 
Also, Khan et al. [14] used coupled homotopy and variational formulation to analyze the aforementioned oscillator. They obtained the first four approximate formulas and concluded that the proposed method observe a good agreement with the exact solution. Moreover, Zuniga [17] employed the Jacobi elliptic functions for the Duffing oscillator and showed that the exact frequency of the system is including the complete elliptic integral of the first kind.

In the current paper, an approach is employed to solve and obtain the analytical approximate solutions of the cubic-quintic Duffing oscillator. This method has a main difference with other traditional harmonic balance methods such that all the errors obtained in the previous approximation are used in the present one. Moreover, to obtain the unknown constants we are dealing with only a set of linear equations which can be easily solved. The comparison that will be done between the presented method, exact solution, coupled homotopy-variational formulation as well as He's energy balance method, will confirm the ability and high precision of this method.

\section{Description of the problem.}

Duffing oscillator with the cubic and quintic terms of nonlinearity is a conservative system and can be defined with the following second-order differential equation $[8,12,17]$

$$
\ddot{x}+\alpha_{1} x+\alpha_{2} x^{3}+\alpha_{3} x^{5}=0,
$$

where, the double dots superscript represents the second differential respect to time $(t)$. The coefficients $\alpha_{1}, \alpha_{2}$ and $\alpha_{3}$ are supposed to get different values. For the case with $\alpha_{1} \neq 0$ and $\alpha_{2}=\alpha_{3}=0$, Eq. (1) represents the simple harmonic vibration of an oscillator with the frequency of $\sqrt{\alpha_{1}}$. If $\alpha_{2} \neq 0$ and $\alpha_{3}=0$, Eq. (1) can be related to Duffing equation with only cubic term of nonlinearity. On the other hand, the quintic Duffing equation is constructed by considering $\alpha_{2}=0$ and $\alpha_{3} \neq 0$. For the other cases, Eq. (1) is named as cubicquintic Duffing equation where $\alpha_{2}$ and $\alpha_{3}$ are not equal to zero.

The initial conditions for Eq. (1) are considered as:

$$
x(0)=A ; x(0)=\frac{d x}{d t}=0 .
$$

\section{Basic idea of the proposed iterative approach.}

To illustrate how the method works, it is considered that the governing differential equation of system is as follows:

$$
\ddot{u}=f(u) ; u(0)=A ; \dot{u}(0)=0,
$$

where $\ddot{u}$ is the second differentiation respects to $t$. It is assumed that $f(u)$ is an odd function. Introducing an independent variable $\tau=\omega t$, Eq. (3) can be rewritten as below:

$$
\omega^{2} u^{\prime \prime}=f(u) ; u(0)=A ; u^{\prime}(0)=0,
$$

where $u$ is the second differentiation respect to $\tau$. Also, $\omega$ is the frequency of system in which should be determined. The solution of Eq. (4) is assumed to be periodic and the simplest form of it that would satisfy the initial conditions is given by the following:

$$
u_{0}(\tau)=A \cos (\tau) ; \omega^{2}=\omega_{0}^{2} .
$$

Substituting Eq. (5) into Eq. (4) and then setting up the coefficient of $\cos (\tau)$ as zero, the parameter $\omega_{0}$ can be determined. Hence, the zero-order approximation of Eq. (3) is as follows:

$$
u_{0}(\tau)=A \cos \left(\omega_{0} t\right)
$$


Eq. (1) is a nonlinear problem and therefore the above solution is not the exact one. Hence, substituting Eq. (6) into Eq. (4), the error for the zero-order approximation derives as:

$$
R_{0}(\tau)=\omega_{0}^{2} u_{0}^{\prime \prime}-f\left(u_{0}\right) .
$$

The above error is kept to be used in the next order of approximation. To obtain the first-order approximation, the following assumption is supposed:

$$
\begin{gathered}
u(\tau)=u_{0}(\tau)+p u_{1}(\tau) ; \omega^{2}=\omega_{0}^{2}+p \omega_{1} . \\
u_{1}(\tau)=B(\cos (\tau)-\cos (3 \tau)) .
\end{gathered}
$$

The coefficient $p$ is the order parameter and takes the values in interval $[0,1]$. In addition, $\omega_{1}$ and $B$ are two unknown constants that will be determined later. In this time, Eq. (8) is substituted into Eq. (4) and then from the governing equation, the coefficients of $p$ are considered as $F_{1}\left(\tau, \omega_{1}, B\right)$. Now, the following relation is considered:

$$
F_{1}\left(\tau, \omega_{1}, B\right)+R_{0}(\tau)=0
$$

Eq. (10) means that in the current method, unlike other traditional harmonic balance methods, the error obtained in the previous step is considered in the present step to increase the accuracy of solution. Equating the coefficients of $\cos (\tau)$ and $\cos (3 \tau)$ to zero in Eq. (10), two linear equations composed of the unknown constants $\omega_{1}$ and $B$ can be achieved. Once the aforementioned constant parameters are obtained, the first-order approximation is written as:

$$
u_{(1)}(\tau)=(A+B) \cos (\tau)-B \cos (\tau) ; \tau=\omega_{(1)} ; \omega_{(1)}^{2}=\omega_{0}^{2}+\omega_{1} .
$$

Substituting Eq. (11) into Eq. (4), the error for the first-order approximation becomes as:

$$
R_{1}(\tau)=\omega_{(1)}^{2} u_{(1)}^{\prime \prime}-f\left(u_{(1)}\right)
$$

Similarly, the above error is kept to be used in the next step. In the following, to obtain the second-order approximation, it is assumed as:

$$
\begin{gathered}
u(\tau)=u_{(1)}(\tau)+p u_{2}(\tau) ; \omega^{2}=\omega_{(1)}^{2}+p \omega_{2} ; \\
\left.u_{2}(\tau)=C(\cos (\tau)-\cos (3 \tau))+D(\tau)-\cos (5 \tau)\right)
\end{gathered}
$$

where $\omega_{2}, C$ and $D$ are three unknown constants which should be determined. Similar to the previous step, Eq. (13) is substituted into Eq. (4) and then the coefficients of $p$ are considered as $F_{2}\left(\tau, \omega_{2}, C, D\right)$. Here, in order to increase the accuracy of the second-order approximation, the error $R_{1}(\tau)$ obtained in Eq. (12) is added to $F_{2}$ and consequently a new equation is obtained as follows:

$$
F_{2}\left(\tau, \omega_{2}, C, D\right)+R_{1}(\tau)=0 .
$$

Equating the coefficients of $\cos (\tau), \cos (3 \tau)$ and $\cos (5 \tau)$ to zero in Eq. (15), three linear equations including the unknown constant parameters $\omega_{2}, C$ and $D$ can be achieved. As the aforementioned constants are obtained, the second-order approximation is written as 


$$
u_{(2)}(\tau)=(A+B+C+D) \cos (\tau)-(B+C) \cos (3 \tau)-D \cos (5 \tau),
$$

where,

$$
\tau=\omega_{(2)} t ; \omega_{(2)}^{2}=\omega_{(1)}^{2}+\omega_{2} .
$$

Similar to the above steps, the higher-order approximations can also be derived. It should be noted that using Eqs. (10) and (15) in the process of solving nonlinear oscillator is the main difference between the current method and other classical harmonic balance techniques. Moreover, these equations (i.e. Eqs. (10) and (15)) lead to a system of linear equations which can be easily solved. The convergence of the proposed method is explained in the Appendix.

\section{Implementation of the method to Duffing oscillator.}

In this section, the approach is used to obtain the approximate solution of Eq. (1). Primarily, introducing an independent variable, $\tau=\omega t$, contributes to transform the mentioned equation to the following:

$$
\omega^{2} x^{\prime \prime}+\alpha_{1} x+\alpha_{2} x^{3}+\alpha_{3} x^{5}=0
$$

where $x^{\prime \prime}$ and $\omega$ are the second differentiation respect to $\tau$, and the frequency of system, respectively. Also, the initial conditions change towards $x(0)=A$ and $x^{\prime}(0)=0$.

\subsection{Zero-order approximation.}

The simplest form of equation in which can satisfy the initial conditions of Eq. (18) is as follows:

$$
x_{0}(\tau)=A \cos (\tau) ; \omega^{2}=\omega_{0}^{2} .
$$

Substituting above equation into Eq. (18), yields

$$
\left(-A \omega_{0}^{2}+\alpha_{1} A+\frac{3}{4} \alpha_{2} A^{3}+\frac{5}{8} \alpha_{3} A^{5}\right) \cos (\tau)+\left(\frac{1}{4} \alpha_{2} A^{3}+\frac{5}{16} \alpha_{3} A^{5}\right) \cos (3 \tau)+\frac{1}{16} \alpha_{3} A^{5} \cos (5 \tau)=0 .
$$

To avoid secular terms in the next step, the coefficient of $\cos (\tau)$ should be identical to zero. Applying this approach, the zero-order approximation (i.e., $\omega_{0}$ ) is obtained as follows

$$
\omega_{0}=\sqrt{\alpha_{1}+\frac{3}{4} \alpha_{2} A^{2}+\frac{5}{8} \alpha_{3} A^{4}}
$$

So, the zero-order analytical approximate solution of Eq. (1) is written as

$$
x_{0}=A \cos \left(\omega_{0} t\right) .
$$

The error for the zero-order approximation obtained as Eq. (23) which will be kept to be used in the next order of approximation:

$$
R_{0}=\left(\frac{1}{4} \alpha_{2} A^{3}+\frac{5}{16} \alpha_{3} A^{5}\right) \cos (3 \tau)+\frac{1}{16} \alpha_{3} A^{5} \cos (5 \tau) .
$$

\subsection{First-order approximation.}

To obtain the first-order approximation, the following assumption is considered

$$
\begin{gathered}
x(\tau)=x_{(0)}(\tau)+p x_{1}(\tau) ; \omega^{2}=\omega_{(0)}^{2}+p \omega_{1} ; \\
x_{1}(\tau)=B(\cos (\tau)-\cos (3 \tau)) .
\end{gathered}
$$

Eq. (24) is substituted into Eq. (18) and then considering the coefficients of the $p$, we obtain a function as $F_{1}\left(\tau, \omega_{1}, B\right)$. According to Eq. (10), we have

$$
F_{1}\left(\tau, \omega_{1}, B\right)+R_{0}(\tau)=0 .
$$


In above equation the coefficients of $\cos (\tau), \cos (3 \tau)$ should be vanished. Therefore, we have two linear equations as follows

$$
\begin{gathered}
\frac{15}{16} \alpha_{3} A^{5} B-A \omega_{1}+\frac{3}{4} \alpha_{2} A^{2} B=0 \\
\frac{5}{16} \alpha_{3} A^{5}+\frac{1}{4} \alpha_{2} A^{3}+8 \alpha_{1} B+\frac{85}{16} \alpha_{3} A^{4} B+6 \alpha_{2} A^{2} B=0 .
\end{gathered}
$$

The solutions of above relations results in:

$$
\begin{gathered}
\omega_{1}=-\frac{3}{16} \frac{A^{3}\left(25 \alpha_{3}^{2} A^{4}+40 \alpha_{3} A^{2} \alpha_{2}+16 \alpha_{2}^{2}\right)}{128 \alpha_{1}+85 \alpha_{3} A^{4}+96 \alpha_{2} A^{2}} \\
B=-\frac{A^{3}\left(5 \alpha_{3} A^{2}+4 \alpha_{2}\right)}{128 \alpha_{1}+85 \alpha_{3} A^{4}+96 \alpha_{2} A^{2}} .
\end{gathered}
$$
ten as

According to Eq. (11), the first-order analytical approximate solution of Eq. (1) is writ-

$$
x_{(1)}=\left(A-\frac{A^{3}\left(5 \alpha_{3} A^{2}+4 \alpha_{2}\right)}{128 \alpha_{1}+85 \alpha_{3} A^{4}+96 \alpha_{2} A^{2}}\right) \cos (\omega t)+\frac{A^{3}\left(5 \alpha_{3} A^{2}+4 \alpha_{2}\right)}{128 \alpha_{1}+85 \alpha_{3} A^{4}+96 \alpha_{2} A^{2}} \cos (3 \omega t),
$$

where $\omega$ is as follows

$$
\begin{gathered}
\omega=\sqrt{\omega_{0}^{2}+\omega_{1}}= \\
=\sqrt{\frac{465 \alpha_{3} A^{4} \alpha_{2}+276 \alpha_{2}^{2} A^{4}+768 \alpha_{2} \alpha_{1} A^{2}+660 \alpha_{3} \alpha_{1} A^{4}+512 \alpha_{1}^{2}+193,75 \alpha_{3}^{2} A^{8}}{340 \alpha_{3} A^{4}+384 \alpha_{2} A^{2}+512 \alpha_{1}}} .
\end{gathered}
$$

Substituting Eq. (30) into Eq. (18), the error is obtained as function $R_{1}(\tau)$.

\subsection{Second-order approximation.}

As in previous steps, for the second-order approximation, it is assumed

$$
\begin{gathered}
x(\tau)=x_{(1)}(\tau)+p x_{2}(\tau) ; \omega^{2}=\omega_{(1)}^{2}+p \omega_{2} ; \\
x_{2}(\tau)=C(\cos (\tau)-\cos (3 \tau))+D(\cos (\tau)-\cos (5 \tau)) .
\end{gathered}
$$

Eq. (32) is substituted into Eq. (18) and then from the consequent equation, the coefficients of $p$ are considered as $F_{2}\left(\tau, \omega_{2}, C, D\right)$. Now, the error $R_{1}(\tau)$ is added to $F_{2}$ and consequently a new equation is obtained as follow:

$$
F_{2}\left(\tau, \omega_{2}, C, D\right)+R_{1}(\tau)=0 .
$$

Equating the coefficients of $\cos (\tau), \cos (3 \tau)$ and $\cos (5 \tau)$ to zero in Eq. (34), one can obtain three linear equations, consequently. By solving these equations, three unknown constants $\omega_{2}, C$ and $D$ can be determined. Given the large amount of space of correlations, we evaded to cite them here. But, their numerical values are reported in the results section. Eventually, the second-order analytical approximation of Eq. (1) is

$$
\begin{gathered}
x_{(2)}=(A+B+C+D) \cos \left(\omega_{(2)} t\right)-(B+C) \cos \left(3 \omega_{(2)} t\right)-D \cos \left(5 \omega_{(2)} t\right) ; \\
\omega_{(2)}^{2}=\omega_{0}^{2}+\omega_{1}+\omega_{2} .
\end{gathered}
$$


5. Results and discussion.

In this section, to assess the analytical approximate solutions achieved by the presented method, the obtained results are compared with the exact solution as well as those of literature. The exact frequency is given by $[13,16]$ :

where:

$$
\omega_{\text {Exact }}=\frac{\pi k_{1}}{2 \int_{0}^{\frac{\pi}{2}}\left(1+k_{2} \sin ^{2} \theta+k_{3} \sin ^{4} \theta\right)^{-\frac{1}{2}} d \theta},
$$

$$
\begin{aligned}
& k_{1}=\sqrt{\alpha_{1}+\alpha_{2} \frac{A^{2}}{2}+\alpha_{3} \frac{A^{4}}{3}} \\
& k_{2}=\frac{3 \alpha_{2} A^{2}+2 \alpha_{3} A^{4}}{6 \alpha_{1}+3 \alpha_{2} A^{2}+2 \alpha_{3} A^{4}} ; \\
& k_{3}=\frac{2 \alpha_{3} A^{4}}{6 \alpha_{1}+3 \alpha_{2} A^{2}+2 \alpha_{3} A^{4}} ;
\end{aligned}
$$

Considering various parameters of system in rank order include as $\alpha_{1}=\alpha_{2}=\alpha_{3}=1$; $\alpha_{1}=5, \alpha_{2}=3, \alpha_{3}=1$; ; and $\alpha_{1}=1, \alpha_{2}=10, \alpha_{3}=100$, the approximate frequencies are obtained and illustrated in Tables $1-3$.

Table 1

\begin{tabular}{|c|c|c|c|c|c|c|}
\hline A & $\omega_{\text {Exact }}$ & Zero-order & First-order & Second-order & Khan et al, [14] & Ganji et al, [8] \\
\hline $\mathbf{0 , 1}$ & 1,0037770 & 1,003774128 & 1,003772940 & 1,003772938 & 1,0031009 & 1,00377306 \\
\hline $\mathbf{0 , 5}$ & 1,1065487 & 1,107502822 & 1,106575472 & 1,106545257 & 1,0877056 & 1,10635650 \\
\hline $\mathbf{1 , 0}$ & 1,5235914 & 1,541103501 & 1,525073610 & 1,523748195 & 1,4456576 & 1,10635650 \\
\hline $\mathbf{5}$ & 19,1815720 & 20,25771458 & 19,37354774 & 19,22145533 & 17,8276787 & 19,608880 \\
\hline $\mathbf{1 0}$ & 75,1776276 & 79,53615530 & 75,97375093 & 75,34539761 & 69,8760834 & 76,889585 \\
\hline $\mathbf{5 0}$ & 1867,5796 & 1976,898075 & 1887,694912 & 1871,836939 & 1735,9103 & 1910,33222 \\
\hline $\mathbf{1 0 0}$ & 7468,8525 & 7906,168540 & 7549,340082 & 7485,889922 & 6942,2827 & 7639,85509 \\
\hline $\mathbf{5 0 0}$ & 186709,59 & 197642,83 & 188721,99 & 187135,59 & 173546,2 & 190984,592 \\
\hline $\mathbf{1 0 0 0}$ & 746836,94 & 790569,89 & 754886,52 & 748540,91 & 694183,44 & 763936,894 \\
\hline
\end{tabular}

Comparison of the frequencies obtained by current approach with the exact solution and the other methods for $\alpha_{1}=\alpha_{2}=\alpha_{3}=1$.

Table 2

\begin{tabular}{|c|c|c|c|c|c|c|}
\hline A & $\omega_{\text {Exact }}$ & Zero-order & First-order & Second-order & Khan et al, [14] & Ganji et al, [8] \\
\hline $\mathbf{0 , 1}$ & 2,2411156 & 2,241107427 & 2,241106482 & 2,241106482 & 2,2402105 & 2,241102478 \\
\hline $\mathbf{0 , 5}$ & 2,3661575 & 2,366762028 & 2,366156024 & 2,366148280 & 2,3434565 & 2,366246867 \\
\hline $\mathbf{1 , 0}$ & 2,7962794 & 2,806243040 & 2,796695889 & 2,796295935 & 2,7566507 & 2,798963393 \\
\hline $\mathbf{5}$ & 20,2164536 & 21,25735167 & 20,39110224 & 20,25141913 & 18,7895069 & 20,64011142 \\
\hline $\mathbf{1 0}$ & 76,1700134 & 80,49844720 & 76,94867693 & 76,33263727 & 70,7962723 & 77,88483819 \\
\hline $\mathbf{5 0}$ & 1868,5568 & 1977,847315 & 1888,654708 & 1872,808907 & 1736,8159 & 1911,314776 \\
\hline $\mathbf{1 0 0}$ & 7469,8296 & 7907,117362 & 7550,299393 & 7486,861400 & 6943,1880 & 7640,837246 \\
\hline $\mathbf{5 0 0}$ & 186710,58 & 197643,7768 & 188722,9500 & 187136,5622 & 173547,11 & 190985,5701 \\
\hline $\mathbf{1 0 0 0}$ & 746837,94 & 790570,8380 & 754887,4839 & 748541,8778 & 694184,38 & 763937,8765 \\
\hline
\end{tabular}


Comparison of the frequencies obtained by current approach with the exact solution and the other methods for $\alpha_{1}=5, \alpha_{2}=3, \alpha_{3}=1$.

Table 3

\begin{tabular}{|c|c|c|c|c|c|c|}
\hline A & $\omega_{\text {Exact }}$ & Zero-order & First-order & Second-order & Khan et al, [14] & Ganji et al, [8] \\
\hline $\mathbf{0 , 1}$ & 1,0397019 & 1,039831717 & 1,039699840 & 1,039697865 & 1,0325994 & 1,039642196 \\
\hline $\mathbf{0 , 5}$ & 2,5247023 & 2,604083332 & 2,535046776 & 2,526416372 & 2,3542032 & 2,554014562 \\
\hline $\mathbf{1 , 0}$ & 8,0100698 & 8,426149772 & 8,080690531 & 8,024286085 & 7,4440041 & 8,176911017 \\
\hline $\mathbf{5}$ & 187,19966 & 198,1186513 & 189,2033240 & 187,6230518 & 174,00040 & 191,4770915 \\
\hline $\mathbf{1 0}$ & 747,32526 & 791,0442465 & 755,3661805 & 749,0266516 & 694,63605 & 764,4279087 \\
\hline $\mathbf{5 0}$ & 18671,400 & 19764,70974 & 18872,63073 & 18713,99620 & 17355,027 & 19098,90111 \\
\hline $\mathbf{1 0 0}$ & 74684,133 & 79057,41585 & 75489,08410 & 74854,52775 & 69418,750 & 76394,13136 \\
\hline $\mathbf{5 0 0}$ & 1867091,6 & 1976424,012 & 1887215,592 & 1871351,538 & 1735457,9 & 1909841,499 \\
\hline $\mathbf{1 0 0 0}$ & 7468365,0 & 7905694,625 & 7548860,931 & 7485404,694 & 6941830,5 & 7639364,525 \\
\hline
\end{tabular}

Comparison of the frequencies obtained by current approach with the exact solution and the other methods for $\alpha_{1}=1, \alpha_{2}=10, \alpha_{3}=100$.

In these tables, the third-order approximate values achieved by coupled homotopyvariational formulation (CHVF-3) [14] as well as the results of He's energy balance method (HEBM) [8] have been also reported. As can be seen, the values of the second-order meets a good agreement with the exact solution. In order to have a better comparison, for example, the errors of the current approach as well as two mentioned methods for the system parameters $\alpha_{1}=5, \alpha_{2}=3, \alpha_{3}=1$ are depicted in Fig. 1 . The correlation of the error is considered as follows:

$$
\text { Error } \%=\frac{\left|\omega_{\text {Exact }}-\omega\right|}{\omega_{\text {Exact }}} \times 100,
$$

where $\omega_{\text {Exact }}$ is obtained from Eq. (37).

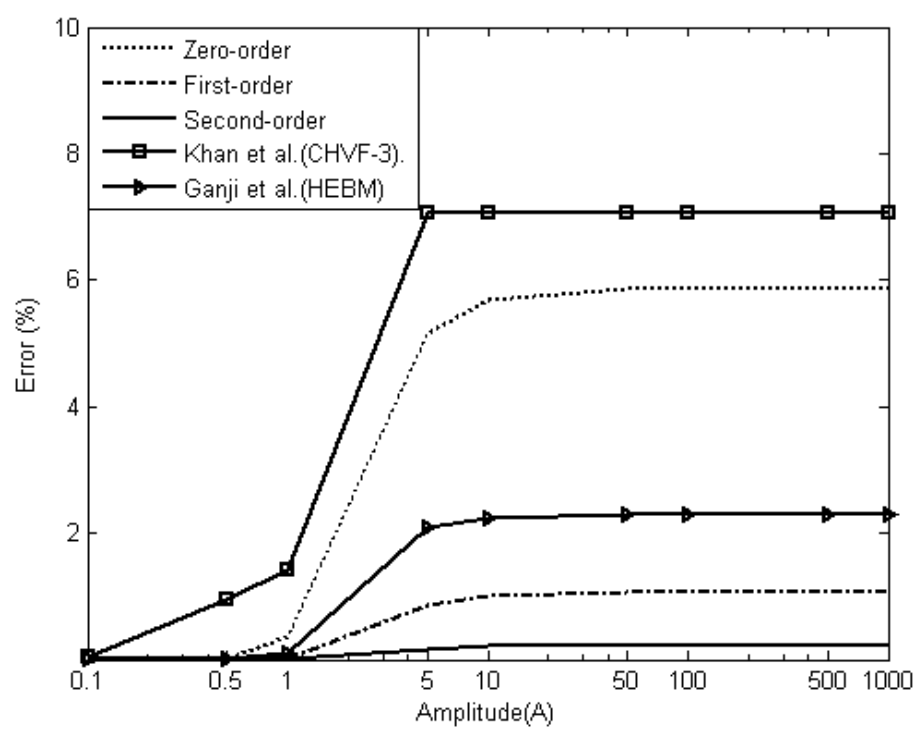

Figure 1. Relative errors for the approximate frequencies respect to amplitude, $\left(\alpha_{1}=5, \alpha_{2}=3, \alpha_{3}=1\right)$. 
Fig. 1 shows that the relative error of the current method decreases with increasing the approximation order. Moreover, with increasing the amplitude (i.e., $A$ ), the error approaches to approximately $7 \%$ and $2,3 \%$ for the CHVF-3 and HEBM, respectively; While it goes toward almost 5,9\%, 1.1\% and $0,2 \%$ for the zero, first and second order approximations, respectively. It is astounding by this figure that merely the first-order approximate solution of the current method leads to accurate results with a relative error less than the third-order coupled homotopy-variational formulation (CHVF-3) as well as He's energy balance method (HEBM). Similar results have been obtained using other system parameters. Hence, it can be concluded that the frequencies achieved by the proposed method can meet the exact ones in the lower-order approximations.

The displacement of the oscillator (i.e., $x(t)$ ) considering various system parameters for large amplitude (here $A=10$ ) is depicted in Figs. 2 and 3.

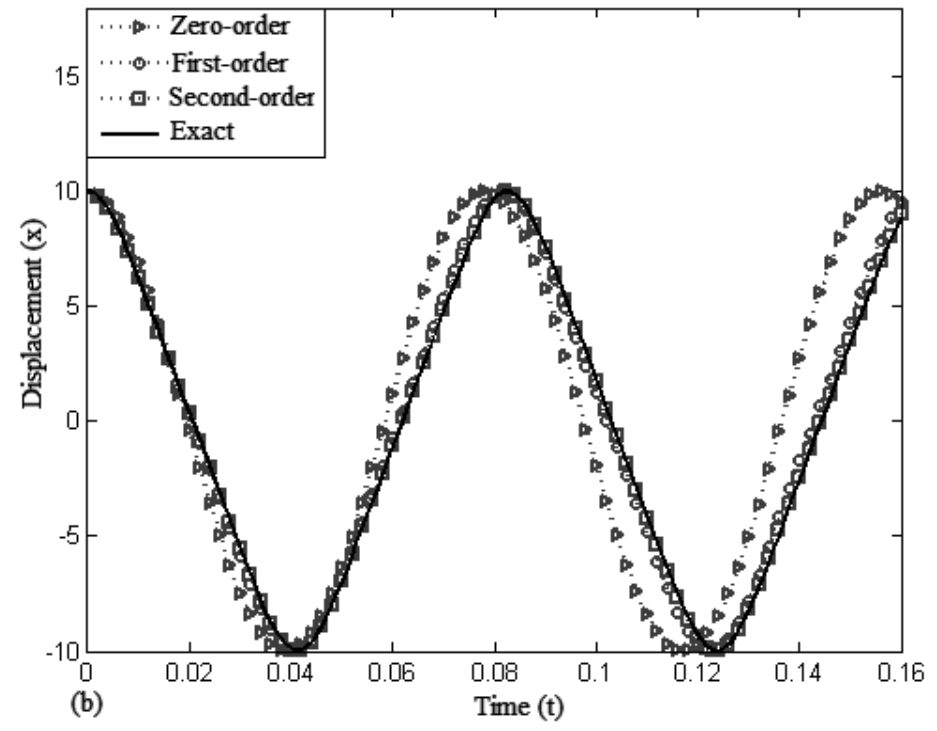

Figure 2. The Displacement of oscillator respect to time for $A=10, \alpha_{1}=5, \alpha_{2}=3, \alpha_{3}=1$

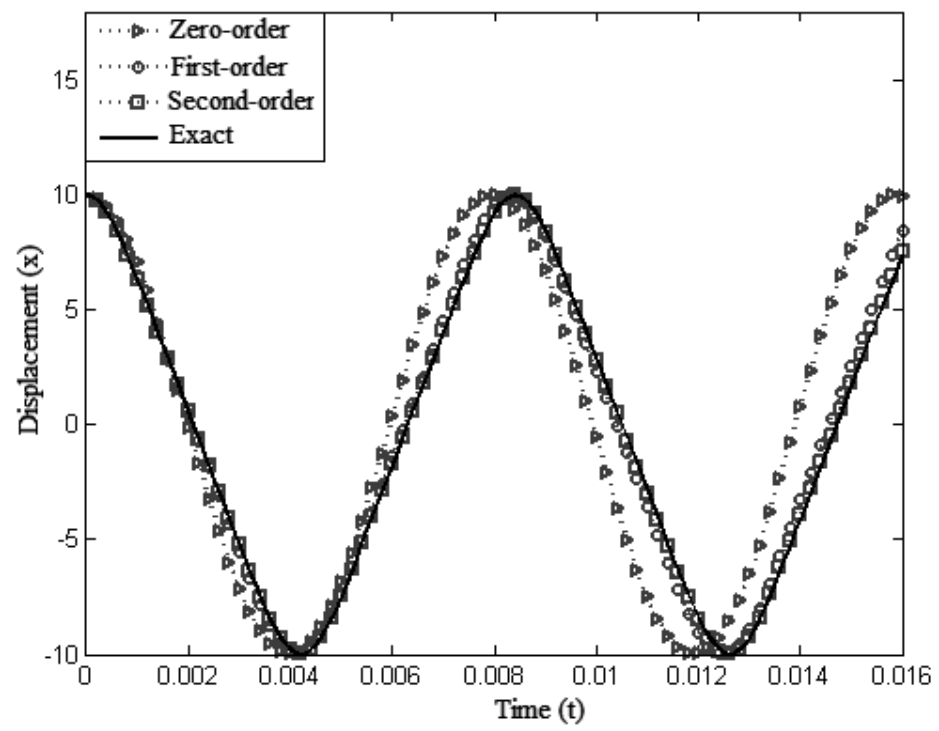

Figure 3. The Displacement of oscillator respect to time for $A=10, \alpha_{1}=1, \alpha_{2}=10, \alpha_{3}=100$ 
These figures reveal that the GRHBM is an accurate method especially in the first and second orders of approximation and has sufficient ability in solving strong nonlinear problems such as current cubic-quintic Duffing oscillator.

The analytical relation obtained in section 4.3, is employed to investigate the effect of nonlinear terms of Eq. (1) on the oscillator frequency. To this end, the coefficients of the cubic and quintic terms (i.e., $\alpha_{2}$ and $\alpha_{3}$ ) are supposed to change from 10 to 50 and 0 to 100 , respectively. The variation of the frequency for various values of amplitude is illustrated in Figs. $4-6$.

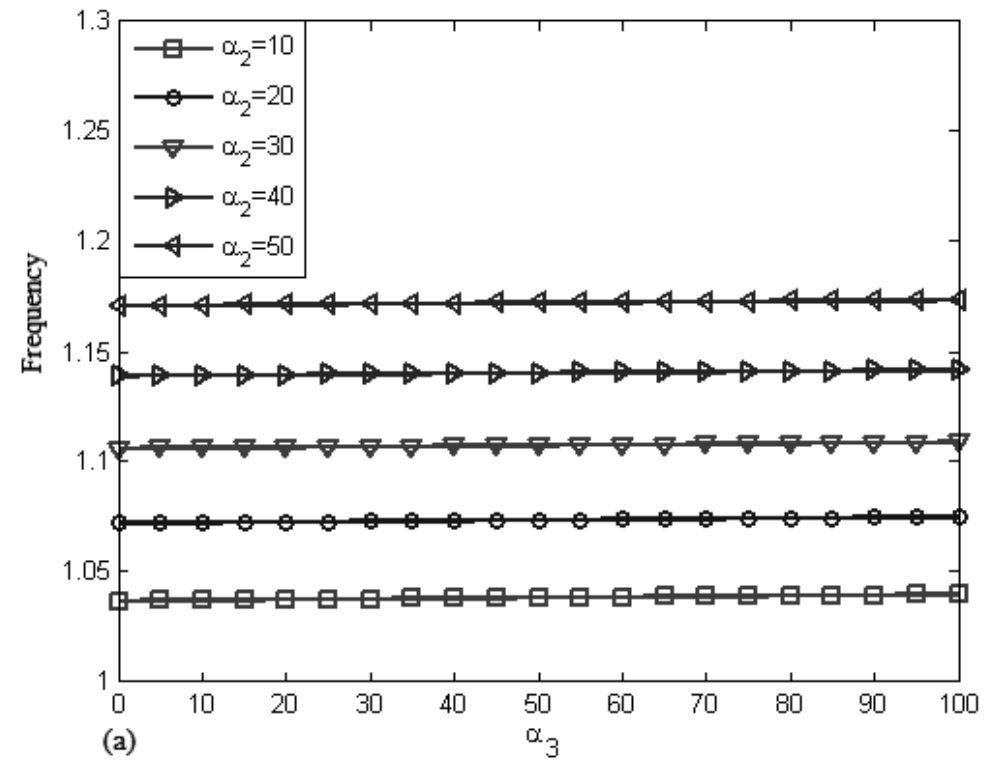

Figure 4. Variation of frequency respect to $\alpha_{3}$ for various values of $\alpha_{2}$ for $A=0,1 ; \alpha_{1}=1$.

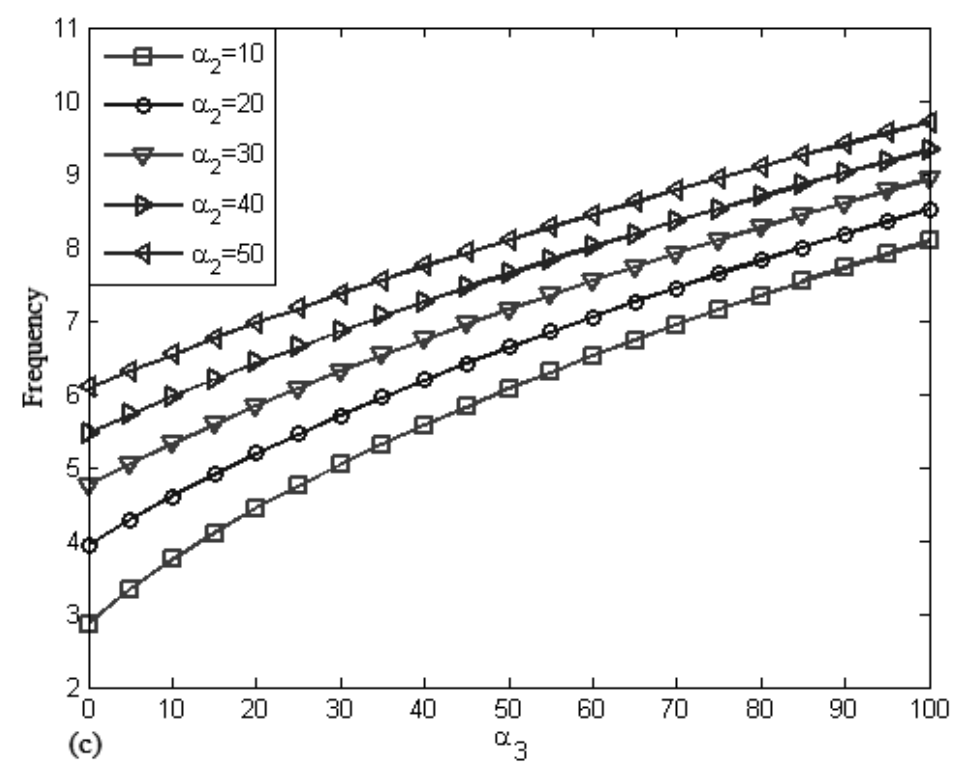

Figure 5. Variation of frequency respect to $\alpha_{3}$ for various values of $\alpha_{2}$ for $A=1, \alpha_{1}=1$. 


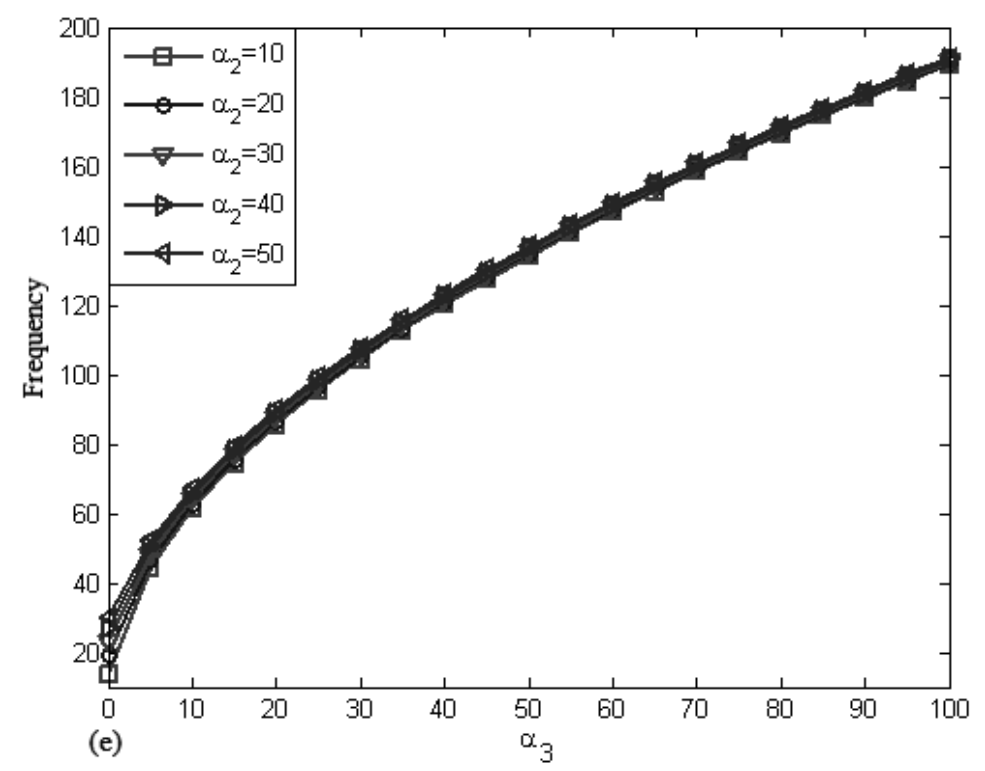

Figure 6. Variation of frequency respect to $\alpha_{3}$ for various values of $\alpha_{2}$

$$
\text { for } A=5, \alpha_{1}=1 \text {. }
$$

Fig. 4 shows that for small amplitude $(A=0,1)$ and a specific value of $\alpha_{2}$, the frequency is approximately constant and is not influenced by the variation of $\alpha_{3}$. Also, this figure shows that for a specific value of $\alpha_{3}$, increasing the $\alpha_{2}$ contributes to increment of frequency. Figs. 5 and 6 reveal that for large amplitudes and any value of $\alpha_{2}$, the frequency increases with increasing $\alpha_{3}$. Moreover, as can be seen in Fig. 6, for large values of amplitude the frequency is only influenced by the variation of $\alpha_{3}$.

\section{Conclusion.}

In this paper, an iterative approach was employed for obtaining the analytical approximate frequencies and displacement of a conservative system comprising an oscillator with cubic and quintic nonlinearities. Using the achieved analytical expressions, the effect of nonlinear terms on the nonlinear frequency was investigated. The results showed that the cubic term is more impressive in the lower amplitudes and its effect gradually vanishes with increment of oscillator amplitude. Also, the quintic term plays a significant role in nonlinear frequency regarding the higher values of amplitude. Moreover, comparing the results in terms of the other methods showed the applicability and accuracy of the proposed method such that it can approach to the exact solution in the lower-order approximations. The mentioned method is very simple to implement and is not restricted to the presence of small parameter in system and its results are valid for a wide range of system parameters along with both small and large amplitudes. Therefore, it can be easily extended and employed for the other strong nonlinear oscillators due to its great potential.

\section{Appendix.}

The proposed method provides a series solution for the problem as follows

$$
u(t)=u_{0}(t)+u_{1}(t)+u_{2}(t)+\ldots=\sum_{i=0}^{\infty} u_{i}(t) .
$$

The above solution converges if there is $0<\beta<1$ such that 


$$
\left\|u_{i+1}(t)\right\| \leq \beta\left\|u_{i}(t)\right\| ; \forall i \geq i_{0} ; i_{0} \in N .
$$

In order to proof, the sequence $\left\{q_{n}\right\}$ is first defined as follows

$$
q_{n}=\sum_{i=0}^{n} u_{i}(t) \quad(n=0,1,2, \ldots)
$$

Then

$$
\left\|q_{n+1}-q_{n}\right\|=\left\|u_{n+1}\right\| \leq \beta\left\|u_{n}\right\| \leq \ldots \leq \beta^{n-i_{0}+1}\left\|u_{i_{0}}\right\| .
$$

For every $j, k \in N, j \geq k>i_{0}$ we have

$$
\begin{gathered}
\left\|q_{j}-q_{k}\right\|=\left\|\left(q_{j}-q_{j-1}\right)+\left(q_{j-1}-q_{j-2}\right)+\ldots+\left(q_{k+1}-q_{k}\right)\right\| \leq \\
\leq\left\|q_{j}-q_{j-1}\right\|+\left\|q_{j-1}-q_{j-2}\right\|+\ldots+\left\|q_{k+1}-q_{k}\right\|=\left\|u_{j}\right\|+\left\|u_{j-1}\right\|+\ldots+\left\|u_{k+1}\right\| \leq \\
\leq \beta^{j-i_{0}}\left\|u_{i_{0}}\right\|+\beta^{j-1-i_{0}}\left\|u_{i_{0}}\right\|+\ldots+\beta^{k+1-i_{0}}\left\|u_{i_{0}}\right\|=\frac{1-\beta^{j-k}}{1-\beta} \beta^{k+1-i_{0}}\left\|u_{i_{0}}\right\| .
\end{gathered}
$$

Consequently

$$
0 \leq \lim _{j, k \rightarrow \infty}\left\|q_{j}-q_{k}\right\| \leq \lim _{j, k \rightarrow \infty} \frac{1-\beta^{j-k}}{1-\beta} \beta^{k+1-i_{0}}\left\|u_{i_{0}}\right\|=0 .
$$

Also, $\lim _{j, k \rightarrow \infty}\left\|q_{j}-q_{k}\right\|=0$ due to have $0<\beta<1$. Sequence $\left\{q_{n}\right\}$ is a Cauchy sequence and according to its definition in the Hilbert space, shows that the solution $\sum_{i=0}^{\infty} u_{i}(t)$ is convergent.

РЕЗЮМЕ. Запропонована ітераційна процедура для отримання наближених розв'язків високого порядку консервативної системи, яка містить осцилятор з відновлювальною силою, що описується третім і п'ятим порядками нелінійності. Запропонований метод аналогічний до класичних методів гармонічного балансу, однак на відміну від них тут похибки, отримані на попередньому кроці, розглядаються на наступному кроці з метою підвищення точності розв'язку. Порівняння результатів 3 результатами, отриманими як точний розв'язок і іншими наближеними аналітичними методиками підтверджує точність методу. Показано, що отримані наближені розв'язки вірні як для малих, так i для великих амплітуд коливань і можуть узгоджуватись 3 точним розв'язком 3 високим рівнем точності при низьких порядках наближень. Далі обговорюється вплив членів третього і п’ятого порядків на основі отриманих аналітичних розв'язків.

1. Semenyuk NP. Nonlinear Deformation of Shells with Finite Angles of Rotation and Low Elastoplastic Strains. International Applied Mechanics. 2015;51:149 - 58.

2. Kochurov RE, Avramov $K V$. Parametric vibrations of cylindrical shells subject to geometrically nonlinear deformation: multimode models. International Applied Mechanics. 2011;46:1010-18.

3. Kirichok IF. Resonant Vibrations and Self-Heating of a Clamped Flexible Thermoviscoelastic Beam with Piezoactuators. International Applied Mechanics. 2014;50:421-9.

4. Mohammadian M, Shariati M. Approximate analytical solutions to a conservative oscillator using global residue harmonic balance method. Chinese Journal of Physics. 2017;55:47 - 58.

5. Mohammadian M. Application of the global residue harmonic balance method for obtaining higher-order approximate solutions of a conservative system. International Journal of Applied and Computational Mathematics. 2017;3:2519-32.

6. Mohammadian M., Akbarzade M. Higher-order approximate analytical solutions to nonlinear oscillatory systems arising in engineering problems. Archive of Applied Mechanics. 2017;87:1317-32. 
7. Khan Y, Mirzabeigy A. Improved accuracy of He's energy balance method for analysis of conservative nonlinear oscillator. Neural Computing and Applications. 2014;25:889-95.

8. Ganji D.D., Gorji M., Soleimani S., Esmaeilpour M. Solution of nonlinear cubic-quintic Duffing oscillators using He's Energy Balance Method. Journal of Zhejiang University SCIENCE A. 2009;10:1263-8.

9. Hermann M., Saravi M., Ebrahimi Khah H. Analytical study of nonlinear oscillatory systems using the Hamiltonian approach technique. Journal of Theoretical and Applied Physics. 2014;8:1 - 8 .

10. Akbarzade M., Farshidianfar A. Application of the Amplitude-Frequency Formulation to a Nonlinear Vibration System Typified by a Mass Attached to a Stretched Wire. International Applied Mechanics. 2014;50:476-83.

11. Mohammadian M. Application of the variational iteration method to nonlinear vibrations of nanobeams induced by the van der Waals force under different boundary conditions. The European Physical Journal Plus. 2017;132:169.

12. Hamdan M.N., Shabaneh N.H. On the large amplitude free vibrations of a restrained uniform beam carrying an intermediate lumped mass. Journal of Sound and Vibration. 1997;199:711-36.

13. Guo Z., Leung AYT, Yang H.X. Iterative homotopy harmonic balancing approach for conservative oscillator with strong odd-nonlinearity. Applied Mathematical Modelling. 2011;35:1717-28.

14. Khan Y., Akbarzade M., Kargar A. Coupling of homotopy and the variational approach for a conservative oscillator with strong odd-nonlinearity. Scientica Iranica A. 2012;19:417-22.

15. Pirbodaghi T., Hoseini S.H., Ahmadian M.T., Farrahi G.H. Duffing equations with cubic and quintic nonlinearities. Computers \& Mathematics with Applications. 2009;57:500-6.

16. Lai SK, Lim CW, Wu BS, Wang C, Zeng $Q C$, He XF. Newton-harmonic balancing approach for accurate solutions to nonlinear cubic-quintic Duffing oscillators. Applied Mathematical Modelling. 2009;33:852-66.

17. Elías-Zúñiga A. Exact solution of the cubic-quintic Duffing oscillator. Applied Mathematical Modelling. 2013;37:2574-9.

From the Editorial Board: The article corresponds completely to submitted manuscript.

Поступила 24.10.2017

Утверждена в печать 30.01.2018 